\title{
The diagnostic potential of glutathione S-transferase (GST) polymorphisms in patients with colorectal cancer
}

\author{
Joanna Waś',B,C, Monika Karasiewicz ${ }^{2, B, D}$, Anna Bogacz, 3,A,C, Karolina Dziekan 3,B, Małgorzata Górska-Paukszta 3,B, \\ Marek Kamiński, ${ }^{5, E}$, Grzegorz Stańk0 $0^{6, B}$, Adam Kamiński ${ }^{7, E}$, Marcin Ożarowski, ${ }^{8, E}$, Bogusław Czerny ${ }^{9, F}$ \\ ${ }^{1}$ Department of Medical Biology, Institute of Cardiology in Warsaw, Poland \\ ${ }^{2}$ Laboratory of International Health, Department of Preventive Medicine, Poznan University of Medical Sciences, Poland \\ ${ }^{3}$ Department of Stem Cells and Regenerative Medicine, Institute of Natural Fibres and Medicinal Plants, Poznań, Poland \\ ${ }^{4}$ Department of Histocompatibility with Laboratory of Genetic Diagnostics, Regional Blood Center, Poznań, Poland \\ ${ }^{5}$ Department of General Surgery and Gastroenterology, Independent Public Clinical Hospital No. 1, Pomeranian Medical University in Szczecin, Poland \\ ${ }^{6}$ Department of General and Hand Surgery, Independent Public Clinical Hospital No. 1, Pomeranian Medical University in Szczecin, Poland \\ ${ }^{7}$ Department of Orthopedics and Traumatology, Independent Public Clinical Hospital No. 1, Pomeranian Medical University in Szczecin, Poland \\ ${ }^{8}$ Department of Biotechnology, Institute of Natural Fibres and Medicinal Plants, Poznań, Poland \\ ${ }^{9}$ Department of Pharmacology and Pharmacoeconomics, Pomeranian Medical University in Szczecin, Poland \\ A - research concept and design; $\mathrm{B}$ - collection and/or assembly of data; $\mathrm{C}$ - data analysis and interpretation; \\ $D$ - writing the article; $E$ - critical revision of the article; $F$ - final approval of the article
}

Address for correspondence

Anna Bogacz

E-mail:aniabogacz23@02.pl

Funding sources

The study was supported by statutory projects from the Institute of Natural Fibres and Medicinal Plants and Pomeranian Medical University in Szczecin (Poland).

Conflict of interest None declared

Received on January 16, 2017 Reviewed on February 15, 2017 Accepted on June 11, 2017

DOI

10.17219/acem/74682

Copyright

Copyright by Author(s)

This is an article distributed under the terms of the

Creative Commons Attribution Non-Commercial License

(http://creativecommons.org/licenses/by-nc-nd/4.0/)

\section{Abstract}

Background. Colorectal cancer (CRC) is one of the most common cancers in the world. Despite improvements in screening for early diagnosis, CRC is one of the leading causes of cancer deaths.

Objectives. The aim of the study was to determine a potential association between the frequency of GSTM1 and GSTT1 null genotypes and the risk of CRC in the Polish population. Moreover, we analyzed the clinical parameters with the glutathione S-transferase (GST) gene polymorphisms in patients with CRC.

Material and methods. The study was conducted on 512 Caucasians, including 279 patients (105 women and 174 men) with CRC. DNA from peripheral blood was extracted and the multiplex polymerase chain reaction (PCR) technique was used for glutathione S-transferase theta (GSTT1) and mu (GSTMT) gene deletion genotyping.

Results. We found no statistically significant differences in the frequency of the GST gene polymorphisms in patients with CRC and controls. The prevalence of the GSTM1*0 variant in the test subjects was higher than in controls (45.9\% vs 42.9\%; $p>0.05$ ). The frequency of the GSTT1*0 variant was also higher in patients with CRC compared to the control population (21.1\% vs 18.9\%; $p>0.05$ ). In addition, the effect of the GSTM1 and GSTT1 polymorphisms on the incidence of CRC was also analyzed. There was a slight, but not statistically significant, increase of the risk of colon cancer for the GSTM1*0 0 and GSTT1*0 variants. Moreover, we examined the GST genotype due to the cancer TNM classification and the location of the primary tumor. Statistically significant differences in the distribution of the GSTT1*0 and GSTT1*1 genotypes in both the stage and the location of the primary tumor were observed.

Conclusions. It is suggested that the GSTT1 polymorphism may have an impact on the severity of the tumor and its location.

Key words: colon cancer, gene polymorphism, GSTM1, GSTT1, multiplex polymerase chain reaction 


\section{Introduction}

Colorectal cancer (CRC) occurs most commonly in developed countries and it is the second most frequent tumor in Poland. The dynamic increase in the number of CRC cases makes Poland a leader among EU countries in the incidence of this disease entity. Europe shows the highest incidence of sporadic CRC worldwide. ${ }^{1}$

Colorectal tumors are often caused by many factors, both genetic and environmental. Several studies present the attempt to use the genotypes of phase II detoxification enzymes - glutathione S-transferase (GST), UDPglucuronosyltransferase (UGT), sulfotransferase (SULT), and N-acetyltransferase (NAT) - as molecular markers of cancer risk. Due to its function, GST was the subject of a number of studies as a potential susceptibility gene for colon cancer. ${ }^{2,3}$ Glutathione S-transferases (GSTs) are a superfamily of multifunctional proteins that play an important role in cellular detoxification. Cytosolic GSTs catalyze the coupling reactions between the nucleophilic reduced glutathione (GSH) and electrophilic forms of drugs, including chemotherapeutic agents (such as platin derivates), environmental pollution and a wide spectrum of xenobiotics. Moreover, GST enzymes are also involved in the detoxification of active metabolites generated during oxidative stress and provide protection against reactive oxygen species (ROS). On the other hand, it has been shown that the activity of GST in tumor cells may be associated with their resistance to chemotherapeutic drugs, because the level of the GST activity in cancer cells is probably significantly increased and may influence the outcome of the treated patients. Due to the limited efficacy of chemotherapy, including the case of gastrointestinal tract cancer, there is a high interest in the individualization of treatment. The GST polymorphisms primarily lead to the reduction of enzymatic activity compared to the wild-type homozygotes. ${ }^{4}$ The glutathione S-transferase theta (GSTT1) and mu (GSTM1) polymorphisms are manifested by the absence of these genes, and the frequency of these variants in the population is dependent on the geographic location. Further, GST isoenzymes are present in most epithelial tissues of the gastrointestinal tract. In addition, clinical studies have shown that the loss of the GSTM1 expression increases the risk of the digestive tract, bladder, lung and skin cancers. According to the European Prospective Investigation into Cancer and Nutrition, studies on the association between the level of polycyclic aromatic hydrocarbon-DNA adduct and dietary antioxidants may be important among GSTM1null individuals..$^{5-8}$

The aim of our study was to evaluate the prevalence of the GSTT1 and GSTM1 genes polymorphisms in patients with CRC and in the control group. Moreover, the clinical value of these findings for cancer therapy was also analyzed.

\section{Material and methods}

The study was conducted on a group of 512 Caucasians, including 279 patients (105 women, 174 men; mean age: $62.49 \pm 10.6$ years) with CRC who were diagnosed and treated in the Clinic Hospital of the Pomeranian Medical University in Szczecin, Poland. In each case, histological studies were the basis for the diagnosis of cancer. The control group consisted of 233 cancer-free subjects ( 80 women, 153 men; mean age: $58.49 \pm 10.6$ years) recruited from the same hospital. All patients were informed about the purpose of the study and submitted written informed consent. The research was conducted with the approval of the Pomeranian Medical University in Szczecin, Poland.

Genetic studies were conducted in the Department of Stem Cells and Regenerative Medicine at the Institute of Natural Fibers and Medicinal Plants in Poznań, Poland. Genomic DNA was obtained from $5 \mathrm{~mL}$ of whole blood using a Qiagen DNA isolation kit according to the manufacturer's protocol (Qiagen, Valencia, USA). DNA concentration was measured with the DeNovix DS-11 Spectrophotometer (DeNovix Inc., Wilmington, USA). Genotyping of the GSTM1 and GSTT1 variants was performed using the polymerase chain reaction (PCR) multiplex technique. For the detection of the GSTM1 and GSTT1 gene deletion, the amplification of CYP1A1 was used as a positive reaction control. A 215 bp fragment for the GSTM1 gene was amplified with the forward and reverse primers as follows: 5'GAACTCCCTGAAAAGCTAAAG-3' and 5'GTTGGGCTCAAATATACGGTG-3'. Forward and reverse primers for the GSTT1 gene amplification were 5'TTCCTTACTGGTCCTCACATC-3' and $5^{\prime}$ TCACCGGATCATGGCCAGCA-3', and the size of the PCR product was $480 \mathrm{bp}$. For the $C Y P 1 A 1$ product amplification (312 bp), the forward primer 5'GAACTGCCACTTCAGCTGTCT-3' and reverse primer 5'CAGCTGCATTTGGAAGTGCTC-3' were used. All primers were synthesized at the Institute of Biochemistry and Biophysics, Polish Academy of Sciences, Warszawa, Poland. Polymerase chain reaction was performed in $20 \mu \mathrm{L}$ reaction mixture containing $100 \mathrm{ng}$ genomic DNA, 2 pmol each primer, $0.25 \mathrm{mM}$ deoxynucleotides (dNTPs), $1 \times$ concentrate PCR buffer, $2 \mathrm{mM} \mathrm{MgCl} 2$ and $1 \mathrm{U}$ Taq polymerase (Novazym, Poznań, Poland). Polymerase chain reaction was performed in a thermocycler PTC-200 (MJ Research, Inc., Waltham, USA) under the following conditions: initial denaturation at $94^{\circ} \mathrm{C}$ for $4 \mathrm{~min}$ and 35 cycles as follows - denaturation at $94^{\circ} \mathrm{C}$ for $30 \mathrm{~s}$, annealing at $60^{\circ} \mathrm{C}$ for $30 \mathrm{~s}$, elongation for $1 \mathrm{~min}$ at $72^{\circ} \mathrm{C}$, and the final elongation step of $5 \mathrm{~min}$ at $72^{\circ} \mathrm{C}$.

The PCR products were visualized on $3 \%$ agarose gel stained with ethidium bromide for $40 \mathrm{~min}$ at $100 \mathrm{~V}$. The products of electrophoresis were evaluated using the system of documentation and computer analysis of the UVI image (KS 4000/Image PC; Syngen Biotech Molecular 
Biology Instruments, Cromwell, USA). GSTT1*0 (null homozygous) and GSTM1*0 (null homozygous) were defined as the absence of the 480 bp or 215 bp PCR product, respectively. At the same time, the presence of the $312 \mathrm{bp}$ fragment for CYP1A1 indicated a successful PCR reaction. Then, SPSS Statistics 17.0 for Windows was utilized for statistical analysis (Chicago, USA). We used the HardyWeinberg equation to calculate the expected genotype frequencies for each polymorphism, which were compared with the observed values using the $\chi^{2}$ test. The expected results are presented with $95 \%$ confidence intervals (CI). Additionally, the correlation analysis between the genotypes and clinical parameters was performed using one-way analysis of variance (ANOVA) test. The values of $\mathrm{p}<0.05$ were considered statistically significant.

\section{Results}

The following cases were observed based on the location of the tumor: sigmoid colon - 96 patients (34.4\%), cecum and ascending colon -78 patients $(28 \%)$, rectum - 52 patients $(18.6 \%)$, transverse colon -39 patients $(14 \%)$, and descending colon - 14 patients (5\%). On the basis of histopathological examination, the following types of cancer were diagnosed: adenocarcinoma G2 in $73.4 \%$ of cases (205 patients), adenocarcinoma G1 in 14.3\% of cases (40 patients), adenocarcinoma G3 in $5.7 \%$ of individuals (16 patients), carcinoma mucinosum in $4.3 \%$ of cases (12 patients), and carcinoma gelatinosum in $2.1 \%$ (6 patients). Moreover, according to the pathological TNM classification (pTNM), we identified preinvasive carcinoma (Tis) in 7 cases $(2.5 \%)$, the tumor invading the muscularis propria (T2) in 40 patients (14.3\%), while in 153 cases (54.8\%), we found the features of T3, with the tumor invading through the muscularis propria into pericolorectal tissues. The infiltration of the tumor to the peritoneum (T4a) occurred in 68 cases $(24.4 \%)$ and in 11 cases (3.9\%), the cancer directly invaded or was adherent to other organs or structures (T4b). A total of 132 patients (47.3\%) had no metastases in regional lymph nodes (N0) and in 89 cases (31.9\%), we observed metastases in 1-3 lymph nodes (N1). Moreover, 58 cases $(20.8 \%)$ had metastases in 4 or more regional lymph nodes (N2). At the time of diagnosis, metastases (M1) were found in 219 patients (78.5\%), and 61 patients
(21.9\%) had no metastasis (M0). Moreover, in 44 cases, the tumor caused intestinal obstruction. In 35 patients, weight loss was a noticeable symptom of the disease, while in the majority $(\mathrm{n}=244)$, weight loss was not observed. The control group consisted of 233 subjects (80 women and 153 men, mean age: $58.49 \pm 10.6$ years) without CRC (symptom-free) and presented with no anemia.

Furthermore, the frequency of the GSTM1 gene polymorphism in CRC patients and the control group was investigated. The analysis of the GSTM1*0 and GSTM1*1 variations was conducted among 279 patients with histologically diagnosed CRC and in 233 control cases. The $\chi^{2}$ test showed no statistically significant differences between the values in the study group and in controls $(\mathrm{p}=0.22)$. The prevalence of the GSTM1 null genotype variant in both groups was similar, and amounted to $45.9 \%$ in the study group and $42.9 \%$ in the control group. The frequency of GSTM1*1 was $54.1 \%$ in the study group and $57.1 \%$ in the control group (Table 1). Furthermore, we determined a risk of colon cancer depending on genotypes. In the case of the GSTM1 null variant, the odds ratio (OR) was slightly elevated $(\mathrm{OR}=1.13,95 \% \mathrm{CI}=0.78-1.63 ; \mathrm{p}=0.28)$. However, there was no significant impact of this polymorphism on the risk of developing colon cancer.

Additionally, the clinical data of patients diagnosed with CRC were compared with the GSTM1 gene genotypes. We selected the clinical stage according to the TNM classification and the primary tumor location. The results are shown in Table 2. In the same group of patients and controls, the GSTT1 gene polymorphism was determined. The $\mathrm{X}^{2}$ test showed no statistically significant difference between the study group and controls $(\mathrm{p}=0.30)$ (Table 3$)$.

The frequency of the GSTT1*0 variant was $21.1 \%$ compared to the control group (18.9\%). The frequency of GSTT1* 1 was $78.9 \%$ in the study group and $81.1 \%$ in controls. The effect of the GSTT1 polymorphism on the incidence of CRC was also analyzed. There was a slightly increased risk of CRC in the case of the GSTT1 null genotype $(\mathrm{OR}=1.15,95 \% \mathrm{CI}=0.73-1.83 ; \mathrm{p}=0.30)$, but not statistically significantly.

Additionally, the clinical data of patients diagnosed with CRC was compared with the GSTT1 gene genotypes. We examined the cancer stage according to the TNM classification and the location of the primary tumor. Statistically significant differences in the distribution of the genotypes

Table 1. The frequency of the GSTM1 genotypes in patients with CRC and controls

\begin{tabular}{|c|c|c|c|c|c|}
\hline \multirow{2}{*}{ Genotype } & Patients & Controls & \multirow{2}{*}{ OR } & \multirow{2}{*}{$95 \% \mathrm{Cl}$} & \multirow{2}{*}{$\mathrm{p}$-value } \\
\hline & observed value n (\%) & observed value n (\%) & & & \\
\hline GSTM1*0 & $128(45.9)$ & $100(42.9)$ & 1.13 & $0.78-1.63$ & 0.28 \\
\hline GSTM1*1 & $151(54.1)$ & $133(57.1)$ & 0.89 & $0.62-1.28$ & 0.28 \\
\hline Total & 279 (100) & $233(100)$ & - & - & - \\
\hline
\end{tabular}

CRC - colorectal cancer; OR - odds ratio; $\mathrm{Cl}$ - confidence interval. 
Table 2. Selected clinical parameters of patients with CRC and the GSTM1 genotype

\begin{tabular}{|l|c|c|c|}
\multirow{2}{*}{ Clinical parameters } & \multicolumn{2}{|c|}{ Genotype n (\%) } & \multirow{2}{*}{ p-value } \\
\cline { 2 - 3 } pT & GSTM1*0 & GSTM1*1 & \\
Tis & & & \\
T1 & $3(1.08)$ & $4(1.43)$ & \\
T2 & - & - & \multirow{2}{*}{0.06} \\
T3 & $19(6.8)$ & $21(7.5)$ & \\
T4 & $68(24.4)$ & $85(30.5)$ & \\
pN & $38(13.6)$ & $41(14.7)$ & \\
N0 & & & \\
N1 & $60(21.5)$ & $72(25.8)$ & \multirow{2}{*}{0.12} \\
N2 & $40(14.3)$ & $49(17.6)$ & \\
\hline M & $28(10)$ & $30(10.8)$ & \\
M0 & & & \multirow{2}{*}{0.15} \\
M1 & $23(8.2)$ & $38(13.6)$ & \\
\hline Tumor location & $105(37.6)$ & $113(40.5)$ & \\
sigmoid colon & & & \multirow{2}{*}{0.64} \\
cecum and ascending colon & $34(12.2)$ & $44(15.7)$ & \\
rectum & $30(10.8)$ & $22(7.9)$ & \\
transverse colon & $21(7.5)$ & $18(6.5)$ & \\
descending colon & $4(1.4)$ & $10(3.6)$ & \\
\hline
\end{tabular}

CRC - colorectal cancer; pT, pN, M - pathological TNM classification of the tumor; Tis - carcinoma in situ; T1- tumor invades the submucosa; T2 - tumor invades the muscularis propria; T3 - tumor invades through the muscularis propria into pericolorectal tissues; T4 - tumor penetrates to the surface of the visceral peritoneum; NO - no regional lymph node metastasis; N1 - metastasis in 1-3 regional lymph nodes; N2 - metastasis in 4 or more regional lymph nodes; $\mathrm{MO}$ - no distant metastasis; M1 - metastasis to distant organs.

in both the stage and the location of the primary tumor were observed. There is a possibility that the GSTT1*0 and GSTT1*1 genotypes may have an impact on the severity of the tumor and its location. The results are shown in Table 4.

\section{Discussion}

It is well-known that GST isoenzymes are responsible for the detoxification of some mutagenic and carcinogenic chemical compounds. For this reason, it is possible that the deletion of the GST gene may be a risk factor in carcinogenesis. Some speculate that the GSTM1 null genotype can be associated with a predisposition to certain cancers, but these reports are inconclusive. On the other hand, predictive markers for the treatment of gastrointestinal cancer are needed. In our study, we assessed the possible role of the GSTM1 and GSTT1 polymorphisms in the development of CRC in the Polish population. We investigated the frequency of the GSTM1 and GSTT1 genotypes and their potential influence on the risk of CRC. Moreover, we also examined the GST genotype with regard to the cancer TNM classification and the location of the primary tumor.

The available data on the frequency of the GSTM1 and GSTT1 gene polymorphisms are quite diverse and depend on the ethnic origin of the analyzed population. Nevertheless, the frequencies of the GSTM1 and GSTT1 genotypes observed in our study are in accordance with those from other European Caucasian populations and consistent with previous studies. ${ }^{2}$ Depending on the author, a lack of the GSTM1 enzymatic activity was observed in 40$60 \%$ of Caucasians, while the GSTT1 null genotype is less frequent and is present in $10-20 \%$ of this population. ${ }^{9}$ In our study, the prevalence of the GSTM1*0 variant in the test subjects was $45.9 \%$ compared to $42.9 \%$ in controls. The frequency of the GSTT1*0 variant was $21.1 \%$ in total cancer cases compared to $18.9 \%$ in the control population. According to Arruda et al., the frequency of the GSTM1 and GSTT1 genes homozygous deletion is quite high among Caucasians and amounts to 55\% for GSTM1 (allele frequency: 0.74) and 18.5\% for GSTT1 (allele frequency: 0.43). ${ }^{10}$ For comparison, in dark-skinned Brazilians, these values were $33 \%$ for the GSTM1 (allele frequency: 0.57 ) and 19\% for the GSTT1 gene (allele frequency: 0.43), whereas in the Amazonian group these values were $20 \%$ for GSTM1 (allele frequency: 0.45) and 11\% for GSTT1 (allele frequency: 0.34). Moreover, the frequency of the GSTM1 and GSTT1 both null genotypes in white- and dark-skinned patients was $11 \%$ (frequency of both alleles: 0.33 ) and 5\% (allele frequency: 0.22), respectively. Importantly, Garte et al. suggest that the highest frequency of the GSTM1*0 homozygotes is in the Caucasian population (0.53), lower in Asians (0.52) and the lowest in Africans (0.26), depending on the origin of the study population. ${ }^{11}$

On the basis of the OR calculation, we found no statistically significant effect of the GSTM1 null (OR $=1.13,95 \%$ $\mathrm{CI}=0.78-1.63 ; \mathrm{p}=0.28)$ and GSTT1 null $(\mathrm{OR}=1.15,95 \%$ $\mathrm{CI}=0.73-1.83, \mathrm{p}=0.30)$ genotypes on the risk of $\mathrm{CRC}$ development. Our findings are consistent with the results obtained by Hezova et al. and Chenevix-Trench et al., where neither of the GSTM1 and GSTT1 polymorphisms showed any associations with CRC. ${ }^{2,12}$ Similarly, no increased risk of developing CRC for both the null variants in a British population study was found. ${ }^{13}$ Most reports indicate that the increased risk is at a lowel, approx. $\mathrm{OR}<2 .{ }^{14,15}$ Still, there are also those who suggest a much

Table 3. The frequency of the GSTT1 genotypes in patients with CRC and the controls

\begin{tabular}{|c|c|c|c|c|c|}
\hline \multirow{2}{*}{ Genotype } & Patients & Controls & \multirow{2}{*}{ OR } & \multirow{2}{*}{$95 \% \mathrm{Cl}$} & \multirow{2}{*}{$p$-value } \\
\hline & observed value $n(\%)$ & observed value $n(\%)$ & & & \\
\hline GSTT1*0 & $59(21.1)$ & $44(18.9)$ & 1.15 & $0.73-1.83$ & 0.30 \\
\hline GSTT1*1 & 220 (78.9) & $189(81.1)$ & 0.87 & $0.55-1.37$ & 0.30 \\
\hline Total & $279(100)$ & $233(100)$ & - & - & - \\
\hline
\end{tabular}

$\mathrm{CRC}$ - colorectal cancer; OD odds ratio; $\mathrm{Cl}$ - confidence interval. 
Table 4. Selected clinical parameters of patients with CRC and the GSTT1 genotype

\begin{tabular}{|c|c|c|c|}
\hline \multirow{2}{*}{ Clinical parameters } & \multicolumn{2}{|c|}{ Genotype n (\%) } & \multirow{2}{*}{$p$-value } \\
\hline & GSTT $1 * 0$ & GSTT1*1 & \\
\hline $\begin{array}{l}\mathrm{pT} \\
\text { Tis } \\
\text { T1 } \\
\text { T2 } \\
\text { T3 } \\
\text { T4 }\end{array}$ & $\begin{array}{c}2(0.7) \\
- \\
7(2.5) \\
35(12.5) \\
15(5.4)\end{array}$ & $\begin{array}{c}5(1.8) \\
- \\
33(11.8) \\
118(42.3) \\
64(22.9)\end{array}$ & 0.02 \\
\hline $\begin{array}{l}\text { pN } \\
\text { N0 } \\
\text { N1 } \\
\text { N2 }\end{array}$ & $\begin{array}{l}27(9.7) \\
12(4.3) \\
20(7.2)\end{array}$ & $\begin{array}{c}105(37.6) \\
77(27.6) \\
38(13.6)\end{array}$ & 0.04 \\
\hline $\begin{array}{l}\text { M } \\
\text { M0 } \\
\text { M1 }\end{array}$ & $\begin{array}{c}16(5.7) \\
43(15.4)\end{array}$ & $\begin{array}{c}45(16.1) \\
175(62.7)\end{array}$ & 0.03 \\
\hline $\begin{array}{l}\text { Tumor location } \\
\text { sigmoid colon } \\
\text { cecum and ascending colon } \\
\text { rectum } \\
\text { transverse colon } \\
\text { descending colon }\end{array}$ & $\begin{array}{c}16(5.7) \\
10(3.6) \\
11(3.9) \\
18(6.5) \\
4(1.4)\end{array}$ & $\begin{array}{c}80(28.7) \\
68(24.4) \\
41(14.7) \\
21(7.5) \\
10(3.6)\end{array}$ & 0.04 \\
\hline
\end{tabular}

CRC - colorectal cancer; pT, pN, M - pathological TNM classification of the tumor; Tis - carcinoma in situ; T1- tumor invades the submucosa; T2 - tumor invades the muscularis propria; T3 - tumor invades through the muscularis propria into pericolorectal tissues; T4 - tumor penetrates to the surface of the visceral peritoneum; NO - no regional lymph node metastasis; N1 - metastasis in 1-3 regional lymph nodes; N2 - metastasis in 4 or more regional lymph nodes; MO - no distant metastasis; M1 - metastasis to distant organs.

larger relationship between the GSTM1/GSTT1 doublenull haplotype and the risk of gastrointestinal cancers. ${ }^{16}$ Furthermore, in our research, no impact of the GSTM1 gene polymorphism on the location and stage of the tumor was noted. Similar results were presented by Gertig et al., Abdel-Rahman et al., Loktionov et al., and Zhu et al. ${ }^{17-20}$ In contrast, Zhong et al. demonstrated an increased frequency of the GSTM1 null genotype in a population of 196 cases with CRC (56.1\%) compared with the control group of 225 individuals (41.8\%). ${ }^{21}$ In their study, the increased evidence of proximal colon tumor in patients with the GSTM1 null genotype was noted. On the other hand, Katoh et al. suggested that the GSTM1 null genotype is a risk factor $(\mathrm{OR}=2.03,95 \% \mathrm{CI}=1.06-3.90)$ among patients with distal colorectal adenocarcinoma, while Wang et al. observed an increased rectal cancer risk $(\mathrm{OR}=1.55,95 \%$ $\mathrm{CI}=1.05-2.30) .{ }^{15,22}$ Furthermore, Katoh et al. indicated that the combined GSTM1 null and GSTT1 null genotypes frequency was similar in adenocarcinoma cases and in controls. ${ }^{22}$ However, they observed a significant increase of the GSTM1 homozygous null genotype in patients with gastric adenocarcinoma (56.8\%) compared to the control group (43.6\%) (OR = 1.70, 95\% CI = 1.05-2.76).

Moreover, in our study we investigated the effect of the GSTT1 gene variants in relation to the tumor location and the pTNM characteristics. Statistically significant differences in the distribution of the GSTT1*0 and GSTT1*1 genotypes in both the stage and the location of the tumor were observed. We noted a high incidence of the GSTM1*1 genotype, especially in patients diagnosed as T3, M1 and N0. The highest percentage was reported in patients with sigmoid colon, cecum and ascending colon and rectum cancers. These relationships were not observed in the British population. ${ }^{13}$ Other suggestions are derived from a meta-analysis where a statistically significant association between the GSTT1 null variant and rectal cancer was observed $(\mathrm{OR}=1.28,95 \% \mathrm{CI}=1.01-1.64) .{ }^{23}$ There were no differences in the distribution of genotypes and the classification according to Duke's staging or histological differentiation, but a strong correlation between the increased risk of CRC and the combined GSTT1 and GSTM1 null genotypes was observed. Similar findings were obtained by Wan et al. for the correlation of the CRC sites and the GSTT1 gene deletion in rectal tumors $(\mathrm{OR}=1.50,95 \%$ $\mathrm{CI}=1.09-2.07$; $\mathrm{p}<0.0001)$, but in a lower level in CRC patients $(\mathrm{OR}=1.33,95 \% \mathrm{CI}=0.94-1.88) .{ }^{24}$ Apart from that, no connection was found between the GSTT1 polymorphism and age, gender, tumor stage and differentiation. Otherwise, the results reported by Zhang et al. indicated that the prevalence of the GSTT1*0 variant was also higher in poorly differentiated cancers than in others. ${ }^{25}$ According to them, the GSTM1*0 genotype was related to the cancer sites, and increased gradually from the right (37\%) to the left colon (44\%) and to the rectum $(60 \%)(\mathrm{p}=0.049)$. Moreover, some authors indicated that the deletion of the GSTT1 gene was more frequent among distal sporadic colorectal adenocarcinoma (SCRAC) cases compared to proximal ones (66.2\% vs 44.4\%; $\left.\chi^{2}=3.97 ; \mathrm{p}<0.05\right)$, and among the elderly compared to younger patients. ${ }^{20}$ Moreover, there are some papers showing that the presence of the GSTT1 null genotype may increase the risk of tumor progression in patients below 70 years of age. ${ }^{12}$ However, other authors do not agree with these proposals. ${ }^{17,26}$ Furthermore, there was no evidence concerning the impact of smoking on the risk of CRC in patients with the GSTM1 null genotype $(\mathrm{OR}=1.2,95 \% \mathrm{CI}=0.3-4.2)$ and for GSTT1 null patients $(\mathrm{OR}=1.1,95 \% \mathrm{CI}=0.3-4.7) .{ }^{17}$

A review of the literature shows that the activity of phase II detoxification enzymes is generally important in the study of cancer epidemiology. Hence, it is worth noting that the polymorphisms of GST enzymes have been widely studied in other cancer cases. For example, a significant increase of the chronic myelogenous leukemia (CML) incidence in patients with the GSTT1 null genotype was observed. ${ }^{27}$ Moreover, the increased toxicity of chemotherapy and reduced survival in children with acute lymphoblastic leukemia (ALL) and the GSTT1 gene deletion was analyzed. There is also a thesis that the GST genes may be associated with the risk of an ALL relapse in childhood, the development of prostate cancer, bladder cancer, and skin malignances. ${ }^{28}$ Moreover, based on meta-analyzes by Wang et al., there is some effect of the GSTT1 null variant on an increasing risk of lung cancer. ${ }^{29}$ 


\section{Conclusions}

In conclusion, it should be considered that the GST polymorphisms leading to reduced activity of the enzyme may be beneficial in the chemotherapy of patients with CRC. However, in our study we did not prove that the GST polymorphisms alone predispose to CRC. Overall, the homozygous GSTM1 null and GSTT1 null genotypes generate slight changes in the risk of developing colon cancer. However, the scale of the risk is greater when the genotype interactions with other factors are considered (e.g., diet, tobacco smoke exposure). It has been known that studies on the effect of the GST polymorphisms are limited by the insufficient amount of data on the phenotypic consequences of this gene deletion in the aspect of CRC. On the other hand, the combined incidence of multiple gene polymorphisms appeared to be important for CRC predispositions and survival. However, we observed differences in the location and stage of the tumor, depending on the GSTT1 genotype in randomly included patients. Due to different reports, there is a question whether the GST polymorphism may be of diagnostic use, and have an impact on the survival of patients and chemotherapy effectiveness.

\section{References}

1. Didkowska J, Wojciechowska U, Zatoński W. Cancer in Poland in 2011 [in Polish]. Warszawa: Polish National Cancer Registry, Department of Epidemiology and Cancer Prevention; 2013.

2. Hezova R, Bienertova-Vasku J, Sachlova M, et al. Common polymorphisms in GSTM1, GSTT1, GSTP1, GSTA1 and susceptibility to colorectal cancer in the Central European population. Eur J Med Res. 2012;17:17.

3. Chirila DN, Popp RA, Balacescu O, et al. GST gene variants in synchronous colorectal cancers and synchronous association of colorectal cancers with other cancers. Chirurgia (Bucur). 2013;108:365-371.

4. Gorukmez O, Yakut T, Gorukmez O, et al. Glutathione S-transferase T1, M1 and P1 genetic polymorphisms and susceptibility to colorectal cancer in Turkey. Asian Pac J Cancer Prev. 2016;17:3855-3859.

5. Sharma N, Singh A, Singh N, et al. Genetic polymorphisms in GSTM1, GSTT1 and GSTP1 genes and risk of lung cancer in a North Indian population. Cancer Epidemiol. 2015;39:947-955.

6. Zamora-Ros R, Barupal DK, Rothwell JA, et al. Dietary flavonoid intake and colorectal cancer risk in the European prospective investigation into cancer and nutrition (EPIC) cohort. Int J Cancer. 2017;140: 1836-1844.

7. Economopoulos KP, Sergentanis TN. GSTM1, GSTT1, GSTP1, GSTA1 and colorectal cancer risk: A comprehensive meta-analysis. Eur J Cancer. 2010;46:1617-1631.

8. Beyerle J, Frei E, Stiborova M, et al. Biotransformation of xenobiotics in the human colon and rectum and its association with colorectal cancer. Drug Metab Rev. 2015;47:199-221.

9. Van Der Logt EM, Bergevoet SM, Roelofs HM, et al. Genetic polymorphisms in UDP-glucuronosyltransferases and glutathione S-transferases and colorectal cancer risk. Carcinogenesis. 2004;25:2407-2415.
10. Arruda VR, Grignolli CE, Goncalves MS, et al. Prevalence of homozygosity for the deleted alleles of glutathione S-transferase mu (GSTMI) and theta (GSTTI) among distinct ethnic groups from Brazil: Relevance to enviromental carcinogenesis. Clin Genet. 1998;54:210-214.

11. Garte S, Gaspari L, Alexandrie AK, et al. Metabolic gene polymorphism frequencies in control populations. Cancer Epidemiol Biomarkers Prevent. 2001;10:1239-1248.

12. Chenevix-Trench G, Young J, Coggan M, et al. Glutathione S-transferase M1 and T1 polymorphisms: Susceptibility to colon cancer and age of onset. Carcinogenesis. 1995;16:1655-1657.

13. Ye Z, Parry JM. Genetic polymorphisms in the cytochrome P450 1A1, glutathione S-transferase M1 and T1, and susceptibility to colon cancer. Teratog Carcinog Mutagen. 2002;22:385-392.

14. Ateş NA, Tamer L, Ateş $C$, et al. Glutathione S-transferase M1, T1, P1 genotypes and risk for development of colorectal cancer. Biochem Genet. 2005;43:149-163.

15. Wang J, Jiang J, Zhao Y, et al. Genetic polymorphisms of glutathione S-transferase genes and susceptibility to colorectal cancer: A case-control study in an Indian population. Cancer Epidemiol. 2011;35:66-72.

16. Martínez C, Martín F, Fernández JM, et al. Glutathione S-transferases mu 1, theta 1, pi 1, alpha 1 and mu 3 genetic polymorphisms and the risk of colorectal and gastric cancers in humans. Pharmacogenomics. 2006;7:711-718.

17. Gertig DM, Stampfer M, Haiman C, et al. Glutathione S-transferase GSTM1 and GSTT1 polymorphisms and colorectal cancer risk: A prospective study. Cancer Epidemiol Biomarkers Prev. 1998;7:1001-1005.

18. Abdel-Rahman SZ, Soliman AS, Bondy ML, et al. Polymorphism of glutathione S-transferase loci GSTM1 and GSTT1 and susceptibility to colorectal cancer in Egypt. Cancer Lett. 1999;142:97-104.

19. Loktionov A, Watson MA, Gunter M, et al. Glutathione-S-transferase gene polymorphisms in colorectal cancer patients: Interaction between GSTM1 and GSTM3 allele variants as a risk-modulating factor. Carcinogenesis. 2001;22:1053-1060.

20. Zhu Y, Deng C, Zhang Y, et al. The relationship between GSTM1, GSTT1 gene polymorphisms and susceptibility to sporadic colorectal adenocarcinoma. Zhonghua Nei Ke Za Zhi. 2002;41:538-540.

21. Zhong S, Wyllie AH, Barnes D, et al. Relationship between the GSTM1 genetic polymorphism and susceptibility to bladder, breast and colon cancer. Carcinogenesis. 1993;14:1821-1824.

22. Katoh T, Nagata N, Kuroda Y, et al. Glutathione S-transferase MI (GSTM1) and TI (GSTT1) genetic polymorphism and susceptibility to gastric and colorectal adenocarcinoma. Carcinogenesis. 1996;17: 1855-1859.

23. Qin X, Zhou Y, Chen Y, et al. Glutathione S-transferase T1 gene polymorphism and colorectal cancer risk: An updated analysis. Clin Res Hepatol Gastroenterol. 2013;37:626-635.

24. Wan H, Zhou Y, Yang P, et al. Genetic polymorphism of glutathione S-transferase $\mathrm{T} 1$ and the risk of colorectal cancer: A meta-analysis. Cancer Epidemiol. 2010;34:66-72.

25. Zhang $H$, Ahmadi A, Arbman G, et al. Glutathione S-transferase $\mathrm{T} 1$ and $\mathrm{M} 1$ genotypes in normal mucosa, transitional mucosa and colorectal adenocarcinoma. Int J Cancer. 1999;84:135-138.

26. Butler WJ, Ryan P, Roberts-Thomson IC. Metabolic genotypes and risk for colorectal cancer. J Gastroenterol Hepatol. 2001;16:631-635.

27. Mondal BC, Paria N, Majumdar S, et al. Glutathione S-transferase $\mathrm{M} 1$ and $\mathrm{T} 1$ null genotype frequency in chronic myeloid leukaemia. Eur J Cancer Prev. 2005;14:281-284.

28. Davies SM, Robison LL, Buckley JD, et al. Glutathione S-transferase polymorphisms and outcome of chemotherapy in childhood acute myeloid leukemia. J Clin Oncol. 2001;19:1279-1287.

29. Wang $Y$, Yang $H$, Wang $H$. The association of GSTT1 deletion polymorphism with lung cancer risk among Chinese population: Evidence based on a cumulative meta-analysis. Oncol Targets Ther. 2015;12: 2875-2882. 